\title{
Probabilistic Analysis of Joint Work Between Pile and Soil
}

\author{
Hongying Jiang ${ }^{1, ~ *, ~ J i a m i n g ~ L ~} \mathbf{u}^{2}$ \\ ${ }^{1}$ School of Civil Engineering, Xijing University, Xi'an, China \\ ${ }^{2}$ Qin and Han Dynasties New Town Development and Construction Group Co., LTD, Xi'an, China
}

\section{Email address:}

1312984559@qq.com (Hongying Jiang), 1106295097@qq.com (Jiaming Lu)

${ }^{*}$ Corresponding author

\section{To cite this article:}

Hongying Jiang, Jiaming Lu. Probabilistic Analysis of Joint Work Between Pile and Soil. American Journal of Civil Engineering. Vol. 5, No. 4, 2017, pp. 231-234. doi: 10.11648/j.ajce.20170504.16

Received: June 30, 2017; Accepted: July 20, 2017; Published: August 2, 2017

\begin{abstract}
Based on the two-dimensional simulation experiment and used the stochastic theory, the regulation of load sharing ratio between pile and soil in composite foundation during construction and consolidation settlement of soil is studied. A simplified probabilistic model is proposed to estimate the load sharing ratio. It provides a reference basis for better study of the predictability of composite foundation and other structural prediction studies based on a variety of materials.
\end{abstract}

Keywords: Load-Sharing Ratio, Consolidation Settlement, Granular Arch Structure, Probability Analysis, Stochastic Theory

\section{Introduction}

Composite foundation is neither an ideal elastic material nor an ideal plastic material. It is a systematic structure made up of diverse materials, in the internal evolution of which there is an effect of both interrelation and synergy. The structural elements that constitute a common feature of the composite foundation are not isolated from each other, It has a complex and singular combined effect. The cushion is one of the core technologies. The flow compensation cushion material can make the soil foundation composite foundation pile always maintain contact, ensure work pile and soil. The cushion is an important component of the composite foundation, the effect of the composite foundation has great influence, therefore, the composite foundation system is a combination of a variety of materials, the macro engineering properties to a great extent by the cushion layer internal fine structure of system state or behavior influence. Its complex physical and mechanical properties are the macroscopic manifestation of its fine structure characteristics. The uncertainty and randomness of the system structure of multi-material combinations are uncontrollable. In recent years, people have studied the mechanical properties of granular materials in terms of micromechanics, although they have been studied a lot, but these studies have not been well combined with engineering applications. Some of the basic problems of particulate matter are still troubling people [1].
Therefore, the mechanical properties of the structure involved with granular materials still call for a lot of research [2-7]. In this paper, based on the two-dimensional simulation experiment and used the stochastic theory, the regulation of load sharing ratio between pile and soil in composite foundation during construction and consolidation settlement of soil is studied. A simplified probabilistic model is proposed to estimate the load sharing ratio. It provides a reference basis for better study of the predictability of composite foundation and other structural prediction studies based on a variety of materials.

\section{Experiment Introduction}

\subsection{Test Content}

The simulation test is scaled down at 1:10, and the measuring device is shown in Figure 1. The size of the test case is $250 \mathrm{~mm}$, height is $150 \mathrm{~mm}$, thickness is $60 \mathrm{~mm}$, and there is a hole at the center of the bottom, that is, the location of the pile head. The pile body is a specially made cement mortar measuring bar with a diameter of $50 \mathrm{~mm}$ and placed vertically at the bottom of the box. The cushion material for good gradation, breccia and particle size greater than $2 \mathrm{~mm}$ more than $50 \%$ of the weight of the particle size greater than $0.5 \mathrm{~mm}$ more than $50 \%$ of the weight of the coarse particles, the fixed thickness is $30 \mathrm{~mm}$, and a wooden hammer is used to tap it till it is dense enough. Pressure sensors are attached 
to the bottom of the pile in order to monitor the force distribution of the pile. And installed outside the bottom of the box are displacement sensors intended to monitor the insertion of the piles as well as four springs with a length of $50 \mathrm{~mm}$ and a stiffness of $\mathrm{k}=0.26$, Inside the bottom of the box there are four miniature pressure cells, fixed with soil, for the purpose of monitoring the changes of pressure under the cushion. A uniformly distributed load is applied from topside, slowly and gently, till given value is reached. The load at each level is set at $0.48 \mathrm{kPa}$. Before the load of the next level is applied, the computer must record the displacement distance and pressure value of the prior level. The data to be taken for calculation must be the average of ten measurements of test data, and the standard errors must be kept under control.

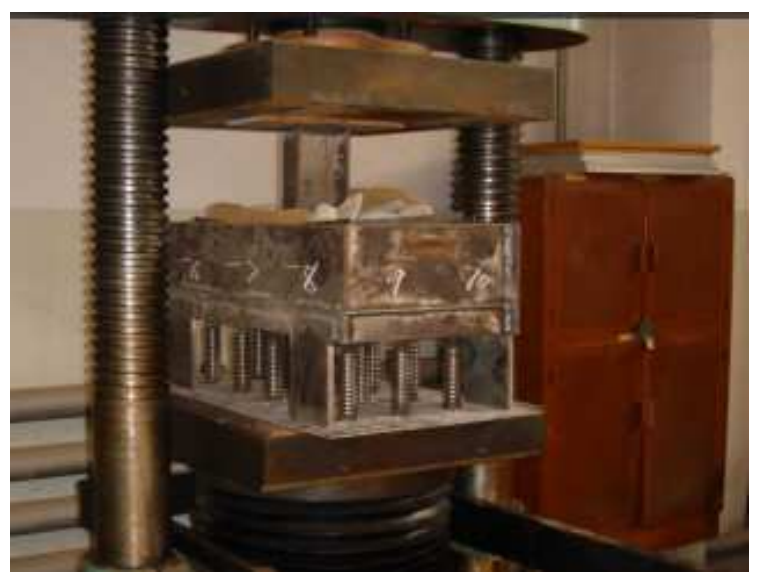

Figure 1. Experimental Device.

\subsection{Analysis of the Experiment Results}

\subsubsection{The Changing Laws of Stress Transfer}

In the experiment, at the beginning of the initial stage, the soil bears greater load. With the increase of the upper load and the pile is inserted into the cushion, the piles comes to play its role, the pile-soil stress ratio takes on increase, the deformation of pile and soil has become synchronous, and the penetration of the pile top to the cushion is basically stable. After that, due to the consolidation settlement of the soil, the soil between the piles will work out and a stress transfer process will occur, and the stress peak will start to transfer to the pile. Then, the pile head began to pierce again, and the stress redistribution occurred.

\subsubsection{Evolution Rules in Cushion Layer}

The cushion is a typical granular structure, internal stress distribution is anisotropic [8]. There is a stress chain in the mattress layer, and then the stress chain generates the scattered arch [9]. The formation of these stress chains leads to uneven stresses, which tend to be concentrated in some local regions and exhibit nonlinear characteristics [6]. When the upper load increases, the cushion layer is compressed and the soil is compressed and the settlement occurs, and the pile head is thrust into the mattress layer, stress transfer, stress redistribution, and the arches formed by granular particles to reassemble. When the soil is consolidated and settled, the cushion and soil re-compression and settlement, the pile head again into the cushion, stress transfer, and stress redistribution. The arching structure consisting of particles are re-assembled. The change in the two processes is the same, but the latter will cause the settlement of the building.

\subsubsection{Shear Resistance in the Cushion Layer}

The cushion is a typical granular structure, internal stress distribution is anisotropic [10]. In the cushion, there exist an effect of granular arch and the phenomenon of jam, and the critical value of internal shear resistance is as follows: $\tau_{f}=\mu \bar{\sigma}$, where $\bar{\sigma}$ is Mean value of normal stress, $\bar{\sigma}=q+\gamma z \quad$ ( $q$ is the load value of the upper part, $\gamma$ is unit volume weight of the cushion material), $\mu=\tan \phi ; \phi$ is the limit value of friction angle inside material particles. When the cushion thickness, with a constant pressure and other conditions, the greater the stiffness and the greater friction angles $\varphi$ of cushion material, the degree of anisotropy is bigger, more easy to take the bulk of the arch, block effect, lateral stress is easy to transfer, easy to pile body stress chain, most stress transfer to the pile. Therefore, the pile-soil stress ratio will be higher [11].

\section{Probability Analysis Model}

The experiment results showed that there is an evolution of load-sharing ratio. The stress ratio of load sharing between pile and soil can be roughly divided into two stages: the first is the construction process, and the second is the consolidation settlement process between the piles. Setting: the load on pile and soil can be superposed. If $s_{c r i}$ is adopted to stand for the critical bearing capacity of the soil, it is assumed that there are $\mathrm{n}$ times for adjusting the increments of a shared load on the soil, so $s_{c r i}$ can be expressed as:

$$
s_{c r i}(f)=\sum_{i=1}^{n} s_{i}\left(f_{i}-f_{i-1}\right)
$$

Where, $s_{i}\left(f_{i}-f_{i-1}\right)$ is the ith increment of load on the soil when the increment of loading is $f_{i}$. When $f<f_{i}$, $s_{i}\left(f_{i}-f_{i-1}\right) \rightarrow 0$, i.e. the increment of load on the soil is zero when there is no increment of loading.

During the adjustment of loading increments, there exist both complexity and randomness. If the probability of $p(n, f)$ is used to characterize the pile-soil load sharing statistics. When the loading amount is $f$, the load sharing condition of pile-soil has changed $n$ times, and each time is independent of each other.

When the load force changes from $f$ to $f+\Delta f$, change takes place in the load-bearing status of the piles and the soil, which has no bearing on $f$ but only on $\Delta f$. Then, when there is a load increment of $\Delta f$, the probability of the soil load sharing with $n$ times is increased by: 


$$
P_{\mathrm{S}}=\lambda_{1} \Delta f+o(\Delta f)
$$

Where, $o(\Delta f)$ is a higher order infinitesimal. $\lambda_{1}$ is the strength of the load transferred to the soil during the adjustment. $\lambda_{1}$ is related to the distance between the pile and the pile, and is related to compactness of cushion material, and is closely related to the cushion material occlusal friction. For the purpose of simplicity, $\lambda_{1}$ is adopted as a constant. At the outset, the cushion is pressed as compact, with the load borne mainly by the soil. As the load increases, the pile head begins to stick into the cushion and the probability that the soil continues to bear the load increment will become random variables subject to negative exponential distribution. Therefore, the probability of transferring load increment for the pile to share is as follows:

$$
P_{\mathrm{P}}=\int \Delta t_{0} \lambda_{2} e^{-\lambda_{2} \Delta f}=\lambda_{2} \Delta f+o(\Delta f)
$$

Where, $\lambda_{2}$ is the strength of load transferred to the piles during the process of loading, identical to $\lambda_{1}$ in the meaning of physics. During the whole process, there is a probability of $P_{\mathrm{S}}+P_{\mathrm{P}}=1$ in any transfer of load value.

Therefore, Kolmogorov equations [12] can be established as follows:

$$
\begin{aligned}
& P_{\mathrm{S}}^{\prime}(f)=-\lambda_{1} p_{\mathrm{S}}(f)+\lambda_{2} p_{\mathrm{P}}(f) \\
& P_{\mathrm{P}}^{\prime}(f)=-\lambda_{2} p_{\mathrm{P}}(f)+\lambda_{1} p_{\mathrm{S}}(f)
\end{aligned}
$$

When the initial conditions $P_{\mathrm{S}}(0)=1, P_{\mathrm{P}}^{\prime}(0)=0$ are met, i.e. without loading, the probability of the soil bearing the load is 1 , and the probability of loading on the pile is 0 . The linear differential equation can be solved as follows:

$$
\begin{array}{r}
P_{\mathrm{S}}(f)=\frac{\lambda_{2}}{\lambda_{1}+\lambda_{2}}+\frac{\lambda_{1}}{\lambda_{1}+\lambda_{2}} e^{-\left(\lambda_{1}+\lambda_{2}\right) f} \\
P_{\mathrm{P}}(f)=\frac{\lambda_{1}}{\lambda_{1}+\lambda_{2}}\left[1-e^{-\left(\lambda_{1}+\lambda_{2}\right) f}\right]
\end{array}
$$

Thus, Formulas (6) and (7) reflect the curve of relation between $P_{\mathrm{S}}(f), P_{\mathrm{P}}(f)$ and load $f$, as shown in Figure 2.

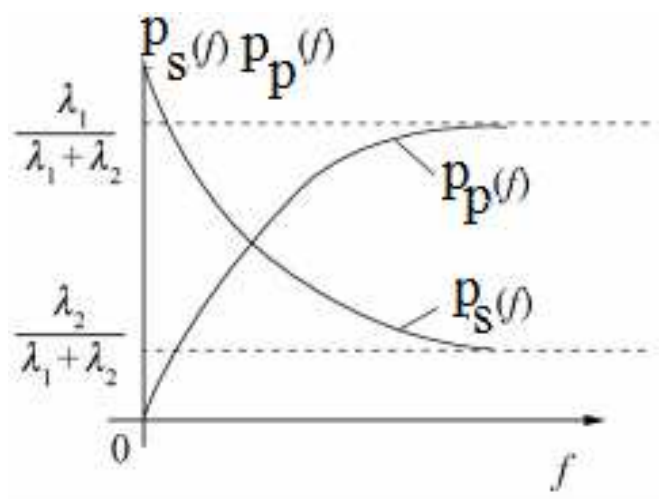

Figure 2. $P_{\mathrm{S}}(f), P_{\mathrm{P}}(f)$ and $f$ load curve.
This curve can reflect the process of continual adjustment of load-sharing ratio between the piles and the soil under the incremental load of construction. After adjustment, when the relative stability is reached, the relative bearing capacity of soil is:

$$
P_{S R}=\frac{\lambda_{2}}{\lambda_{1}+\lambda_{2}}
$$

Therefore, when the state of the transition reaches a relatively steady state, the absolute carrying capacity of the soil is:

$$
P_{S A}=\lambda_{1} P_{S R}
$$

And the bearing capacity of the pile is:

$$
P_{P A}=1-P_{S R}
$$

Therefore, when the state is stabilized, the ratio of the ultimate load sharing between the soil to the pile is mainly related to $\lambda_{2}$ (strength of the load shared by the pile). And the second stage, that is, the repetition of the first stage.

\section{Case Analysis}

In the two stage, the and $\lambda_{2} \lambda_{1}$ value is determined by means of the maximum likelihood estimate [11], where $\lambda_{1,2}=E[N(f)] / \Delta f$ is taken, where $E[N(f)]$ is the average of the load variation on the soil within the $\Delta f$ range.

Here, the cushion thickness is $300 \mathrm{~mm}$, and the test results are shown in Figure 3 , The $\lambda$ mean value of $\bar{\lambda}_{2}=0.95$ are $\bar{\lambda}_{1}=1.02$.

According to the calculation results of (6), (7) and (8), see Figure 3.

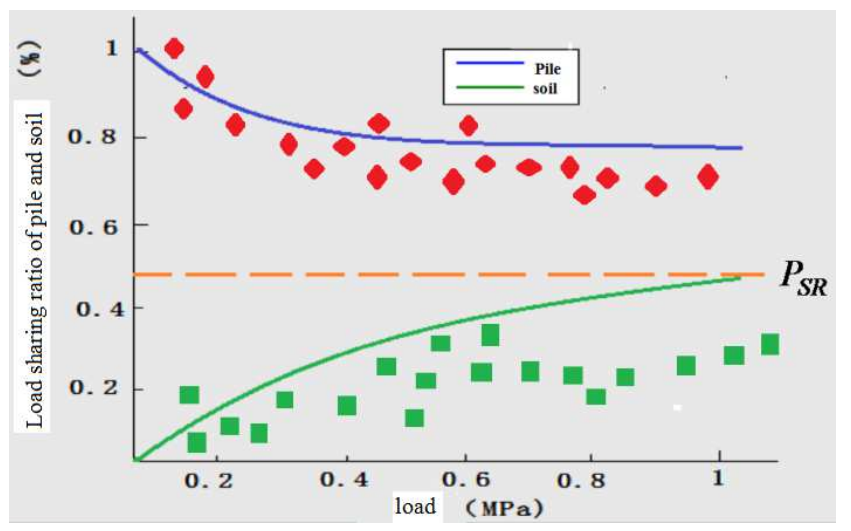

Figure 3. The pile-soil load share ratio.

The red dot (pile), green dot (soil) is the test results and the green line (pile), blue line (soil) is the result of the calculation. Thus, the model estimation is close to the experimental results. 


\section{Conclusion}

Through the microscopic analysis of the evolution law of the load sharing ratio of the pile and soil in two stages, the main characteristics are obtained:

1) In the two adjustment process, the stress ratio has an evolution law, and the statistic of the bearing capacity can be described by the Poisson process;

2) when the pile penetration, the cushion has response process: continuous self organization to arch and stress transfer.

There are many complicated and stochastic factors in the system structure of multi material combinations with granular materials, which brings many difficulties to the theoretical research. Therefore, extensive and in-depth experimental and theoretical studies are needed.

\section{References}

[1] Ebrahim Alizadeh, François Bertrand, Jamal Chaouki. Development of a granular normal contact force model based on a non-Newtonian liquid filled dashpot [J]. Powder Technology 2013 (237):202-212.

[2] Jiang Yan. Research the cushion of rigid pile composite foundation [D]. Zhengzhou: College of civil engineering, Zhengzhou University, 2010.

[3] Robert Zalewski, Mariusz Pyrz. Expermental study and modeling of polymer granular structures submitted to internal underpressure [J]. Mechanics of Materials 57 (2013) 75-85.
[4] G. Marketos, C. O'Sullivan. A micromechanics-based analytical method for wave propagation through a granular material $[\mathrm{J}]$. Soil Dynamics and Earthquake Engineering 45 (2013) 25-34.

[5] Hou Mingxun The Test on the Stress Grid of Granular Material and Characteristic Research on Arching Effect [D]. South China University of Technology 2016.

[6] Zhao Bo, Jimmy Wang. Influence of different arrangement of double row piles on retaining performance based on soil arching effect $[\mathrm{J}]$ Chinese and foreign highway 20162 (36) $11-15$.

[7] Yang Guanghua, Li Deje, Guan Dashu. Optimization deign of rigid pile composite Foundation [J]. Chinese Journal of Rock Mechanics and Engineering. 2011, (04):818-825.

[8] I. Albert, J. G. Sample, A. J. Morss, S. Rajagopalan, A.-L. Baraba'si, and P. Schiffer. Granular drag on a discrete object: Shape effects on jamming [J]. Physical Review E, 2001 (64): 061303-1-061303-3.

[9] Ning Guo, Jidong Zhao. The signature of shear-induced anisotropy in granular media $[\mathrm{J}]$. Computers and Geotechnics 2013 (47):1-15.

[10] Jacco H, Snoeijer, Martin van Hecke, et al. Force and weight distributions in granular media: Effects of contact geometry [J]. Physical Review E, 2003 (64): 030302-1-030302-5.

[11] Zhou Aijun, Li Bing, experimental research and finite element analysis of CFG pile composite foundation cushion [J]. rock and soil mechanics, 2010, 6 (31) 1803-1808.

[12] Liu Jiakun. Application of stochastic process [M]. Beijing: Science press. 2000:47-52. 